\title{
Plasma Homocysteine is Not Related to the Severity of Microangiopathy in Secondary Raynaud Phenomenon
}

\author{
Vincenzo Jacomella, Monika Wasila, Marc Husmann, Gabriela Gitzelmann, Thomas Meier and \\ Beatrice Amann-Vesti*
}

Clinic for Angiology, University Hospital, Zurich, Switzerland

\begin{abstract}
Introduction: The role of elevated homocysteine in primary and secondary Raynaud phenomenon (RP) and in patients with atherosclerosis has been reported controversially. In secondary RP due to connective tissue disease specific alterations of nailfold capillaries might be present. An association between these microvascular changes and homocysteine has been suggested.

Aim: The aim of this study was to determine whether homocysteine level differs between patients with primary and secondary RP and to test the hypothesis that homocysteine or other cardiovascular risk factors are associated with specific features of microangiopathy in secondary RP.

Patients and Methods: Eighty-one consecutive patients with RP referred for vascular assessment were studied by nailfold capillaroscopy. Homocysteine, C-reactive protein and cholesterol were measured and other cardiovascular risk factors and comorbidities assessed.

Results: Homocysteine, C-reactive-protein and cholesterol levels did not differ between patients with primary $(\mathrm{n}=60)$ and secondary RP $(n=21)$. Likewise, no differences in the prevalence of cardiovascular risk factors and comorbidities were found. In secondary RP no correlation was found between microvascular involvement and homocysteine or C-reactive protein.

Conclusion: Plasma homocysteine is not different in patients with either primary or secondary RP and is therefore not a marker for the distinction of these diseases. The extent of microvascular involvement in secondary RP does not correlate with plasma homocysteine.
\end{abstract}

Keywords: Raynaud phenomenon, homocysteine, nailfold capillaroscopy, microangiopathy.

\section{INTRODUCTION}

Raynaud phenomenon results from pathological vasospastic response resulting in acral ischemia. The exact pathomechanism remains unclear. Homocysteine (Hcy) produces vascular endothelial damage and modifies circulating mediators of vasomotion. The effect of Hcy might be directly toxic to endothelial cells of the vessel leading to an activation of signal transduction pathways with production of superoxide radicals resulting in inflammation and apoptosis [1-8]. Superoxide radicals as well as Hcy may directly alter nitric oxide release in the endothelium and hence decrease vasodilatory capacity and promote vasoconstriction [1, 9-11]. Therefore a potentially relevant role for homocysteine in the pathogenesis of RP has been proposed. Different small studies reported increased levels of Hcy in secondary and primary RP [12-16]. Hcy may confer a mild risk alone, but it increases the risk of vascular disease in association with other cardiovascular risk factors such as arterial hypertension, dislipidemia and inflammation which all promote vascular lesions. Plasma Hcy levels $>10 \mu \mathrm{mol} / 1$

\footnotetext{
*Address correspondence to this author at the Clinic for Angiology, University Hospital Zurich, 8091 Zurich, Switzerland; Tel: ++41 44255 2671; Fax: ++41 442554375 ; E-mail: Beatrice.amann@usz.ch
}

are considered cytotoxic and are found in 5 to $10 \%$ in the general population and in up to $40 \%$ of patients with vascular disease and cardiovascular risk factors [17]. Elevated levels of Hcy might be involved not only in atherogenesis but in the development of microvascular changes in rheumatic disease [18]. In contrast to primary RP, different diseases with secondary RP such as rheumatoid arthritis, systemic lupus erythematosus (SLE), systemic sclerosis ( $\mathrm{SSc}$ ), the antiphospholipid syndrome (APS), and systemic vasculitides have been associated with vascular inflammation, altered angiogenesis, and increased cardiovascular morbidity and mortality. Therefore, Hcy could be the common pathway of classical risk factors on one side, and inflammation on the other side.

A recent study reported that Hcy level correlates with the progression of microvascular changes found by nailfold capillaroscopy in patients with secondary RP [19]. Nailfold capillaroscopy has been accepted as one of the most valuable diagnostic and predictive tool for connective tissue disease, especially systemic sclerosis and CREST and specific microvascular changes might even precede the development of serological markers [20]. Reports on hyperhomocysteinemia in patients with secondary RP in relation to microvascular changes are conflicting and scarce, and the assessed number of patients relatively small. We therefore 
tested the hypothesis that hcy plasma levels are associated with microvascular changes in secondary RP and would be different in patients with primary RP. The goal of the present study was to assess Hcy and microvascular alterations as well as cardiovascular risk factors in patients suffering from RP.

\section{METHODS}

\section{Patient Selection and Study Design}

The study was conducted from 2002 to 2005 at a tertiary referral centre as a prospective, non-randomised, singlecentre study. Plasma levels of cholesterol, homocysteine, Creactive protein, cardiovascular risk factors and comorbidities as well as patterns of nailfold capillaroscopy (NC) were assessed in patients with primary and secondary RP. To distinguish between primary and secondary RP we used the clinical criteria according to the American College of Rheumatology. Exclusion criteria were any history of liver diseases, cancer, or drugs that could interfere with Hcy. The patients underwent physical examination, nailfold capillaroscopy of all fingers and serological testing as part of the assessment for RP.

Hospital-based electronic charts were consulted to check follow-up to determine differentiation between primary and secondary RP in initially unclassified cases.

The local ethical committee had approved the study and all patients gave written informed consent.

\section{Cardiovascular Risk Factors}

Arterial hypertension was defined as systolic blood pressure $>140 \mathrm{mmHg}$, and diastolic blood pressure $>90 \mathrm{mmHg}$ measured in triplicate and after a resting period of 20 minutes; diabetes as fasting blood sugar $>7 \mathrm{mmol} / 1$; hypercholesterolemia as fasting total serum cholesterol $>5 \mathrm{mmol} / 1$; smoking was defined as current smoker or smoking cessation in the last ten years with at least ten pack years of smoking.

\section{Homocysteine, C-Reactive Protein, Cholesterol and Serological Markers}

The blood samples were taken after an overnight fast between 8 am and noon for determination of plasma levels of homocysteine (Hcy), C-reactive protein, cholesterol, antinuclear antibodies (ANA), anti-centromere antibodies (ACA), anti-scleroderma 70 antibodies (anti-Scl 70) and anticitrullinated peptide antibodies. Hcy and CRP were measured by using a chemiluminescence-enzymeimmunoassay method (Institute for Clinical Chemistry, University Hospital Zurich, Switzerland), (5 to $13.5 \mu \mathrm{mol} / 1$, norm $<5$ $\mathrm{mg} / \mathrm{l}$ ), cholesterol by usual enzyme test (CHOD-PAP method), (norm $<5 \mathrm{mmol} / \mathrm{l})$. Immunofluorescence method was used to measure ANA levels (norm $<1: 10$ ), nephelometry method for anti-citrullinated peptide antibodies $(<20 \mathrm{E} / \mathrm{ml})$, Dot-Blot method for positive anti-scl 70 antibodies and fluorescence enzyme immuno assay method for ACA levels (norm $<10 \mathrm{U} / \mathrm{ml}$ ).

\section{Nailfold Capillaroscopy}

Nailfold capillaroscopy (NC) had been performed as described [20]. We used an incident light fluorescence microscope (Leica, Heerbrugg, Switzerland) with a mercury vapor lamp (Leica, Heerbrugg, Switzerland) mounted on a heavy support (Foba, Zurich, Switzerland) with a 3-CCD video camera (DXC-930P, Sony, Tokyo, Japan) with a camera adaptor and sensitivity set on automatic control (CMA-D2, Sony), a video timer (VTG-22) and scale marker (IV-600; both from For-A-Company, Tokyo, Japan), a video monitor (Picture Monitor model PM 171T, Ikegami Tsushinki, Tokyo, Japan) and a Sony DVCAM Recorder/Player DSR- 45P. The microscope is equipped with 1.0/0.04, 2.5/0.08, 6.3/0.20 and 10/0.25 planar objectives (Leica), which allow a magnification of x24, x62, $\mathrm{x} 165$, and $\mathrm{x} 240$, respectively, on the monitor.

The nailfold of all ten fingers were examined. To improve the image resolution, a drop of immersion oil had been deposited on the nailfold bed. The following parameters of the nailfold capillaries were evaluated from the videotape:

- Irregular architecture defined as capillaries not in one row, small areas $(<500 \mu \mathrm{m})$ with missing capillaries next to areas with clusters of capillaries.

- Avascular fields defined as loss of capillaries in a field of at least $500 \mu \mathrm{m}$.

- Dilatation of capillaries defined as arterial conduit side greater than $15 \mu \mathrm{m}$ and vein side greater than $20 \mu \mathrm{m}$.

- Giant capillaries defined as an apical diameter of greater than $50 \mu \mathrm{m}$ with or without hemorrhages.

Microvascular alterations were classified into "none", "early", and "active" and "late" patterns representing morphological features in connective tissue disease according to Cutolo et al. [21]. Regular architecture, giant capillaries, few haemorrhages, and no avascular fields represent the "early" pattern. Irregular architecture, frequent giant capillaries and/or haemorrhages are features of the "active" pattern that is considered highly specific for secondary RP. The "late" pattern representing the late stage of disease shows few giant capillaries, haemorrhages and in addition to them avascular fields. NC was performed and evaluated by a vascular technician and reviewed independently by an experienced physician.

\section{Statistics}

Continuous variables were presented as means and standard deviation or median with interquartile ranges as appropriate due to skewed distribution. Categorical variables were expressed as percentages. Qualitative data were compared by the Fischer's exact test. Quantitative data were compared using Student's t test and Mann-Whitney U test, the Kruskal-Wallis test with Bonferroni Dunn corrections. The statistical software package Stat View 5.0 was used to analyse the data. All p values are two-sided. Differences were considered statistically significant if $\mathrm{p}<0.05$.

\section{RESULTS}

\section{Baseline and Clinical Characteristics}

Clinical characteristics of the 81 patients (77\% females) are shown in Table 1. Primary RP was diagnosed in 60 (74\%) patients and $21(26 \%)$ had a secondary RP. Another ten patients with secondary RP were excluded from the 
analysis due to different underlying diseases (two patients had a traumatic finger artery occlusion, two patients an embolic occlusion of a digital artery, two patients had been diagnosed with thrombangitis obliterans and four were unclassified). In female patients the prevalence of secondary $(86 \%)$ and primary $(73 \%) \mathrm{RP}$ was not different. Although the patients with secondary RP tended to be older than the patients with primary RP this difference was not significant. Likewise, there were no significant differences for the presence of cardiovascular risk factors and co-morbidities between the two groups. There was no difference in Hcy and CRP between the two groups (Fig. 1). Table 2 shows the disease characteristics of the group with secondary RP with a $50 \%$ prevalence of unclassifiable secondary RP, followed by CREST (29\%) and systemic sclerosis (19\%).

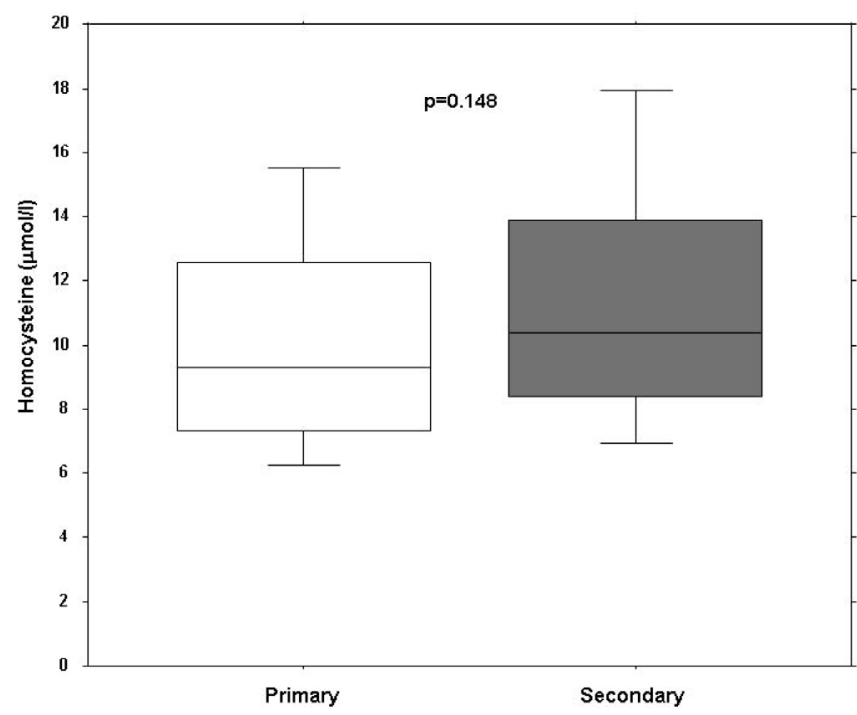

Fig. (1). Box plots of homocystein plasma serum levels in patients with primary and secondary Raynaud phenomenon.
Table 2. Disease Characteristics of Patients with Secondary RP $(n=21)$

\begin{tabular}{|c|c|}
\hline Systemic Sclerosis, $\mathrm{n}(\%)$ & $4(19)$ \\
\hline CREST, $\mathrm{n}(\%)$ & $6(29)$ \\
\hline Mixed Connective tissue disease, $\mathrm{n}(\%)$ & $1(5)$ \\
\hline Sjögren Syndrome, $\mathrm{n}(\%)$ & $1(5)$ \\
\hline Unclassified, n (\%) & $14(67)$ \\
\hline
\end{tabular}

In patients with secondary RP $90 \%$ showed positive ANCA serum levels. $13 \%$ patients presented high anticentromere antibodies, $31 \%$ with positive rheumatoid factors and $27 \%$ with positive anti-scleroderma-70 antibodies.

\section{Microvascular Patterns in Primary and Secondary Raynaud Phenomenon}

As expected, in patients with primary RP only minor changes, mainly capillary tortuosity, of nailfold capillaries have been found. In contrast, there were major structural changes in patients with secondary RP. In $29 \%$ of patients giant capillaries, in $60 \%$ irregular architecture and in $38 \%$ haemorrhages were described (Table 3). This microvascular alterations translated into the definition of Cutolo et al. was as follows: Patients with primary RP $(n=60)$ did not show signs of structural microangiopathy and were hence classified with "no microangiopathy". From the 21 patients with secondary RP had 43\% "early", 19\% "none", $10 \%$ "active", and 28\% "late" pattern.

Median serum level of Hcy was $9.3 \mu \mathrm{mol} / \mathrm{l}$ (IQR 7.4 to $12.6 \mu \mathrm{mol}$ ) in patients with primary and $10.2 \mu \mathrm{mol} / \mathrm{l}$ (IQR 8.2 to $14.7 \mu \mathrm{mol})$ in patients with secondary RP $(\mathrm{p}=0.15)$.

There were no significant differences in Hcy levels in relation to microvascular patterns (Fig. 2). There was no

Table 1. Clinical Characteristics of Patients with Primary and Secondary RP

\begin{tabular}{|c|c|c|c|}
\hline & $\underset{n=60}{\text { Primary RP }}$ & $\underset{n=21}{\substack{\text { Secondary RP } \\
n=2}}$ & $\boldsymbol{P}$ \\
\hline Mean Age, yrs (range) & $47(14-84)$ & $51(21-83)$ & n.s \\
\hline Females, n (\%) & $44(73)$ & $18(86)$ & n.s \\
\hline Mean Body-Mass Index, $\mathrm{kg} / \mathrm{m}^{2}$ (range) & $22(17-32)$ & $24(18-32)$ & n.s \\
\hline Hypertension, $\mathrm{n}(\%)$ & $7(12)$ & $5(19)$ & n.s \\
\hline Diabetes mellitus, n (\%) & $4(7)$ & $1(5)$ & n.s \\
\hline Hypercholesterolemia, mmol/l (SD) & $5.3( \pm 1.1)$ & $4.3( \pm 0.9)$ & n.s \\
\hline Smoking, $\mathrm{n}(\%)$ & $21(35)$ & $9(43)$ & n.s \\
\hline Coronary artery disease, $\mathrm{n}(\%)$ & $0(0)$ & $1(5)$ & n.s \\
\hline Cerebrovascular disease, n (\%) & $2(3)$ & $1(5)$ & n.s \\
\hline Peripheral arterial disease, $\mathrm{n}(\%)$ & $4(7)$ & $2(10)$ & n.s \\
\hline Systolic Blood Pressure, mmHg (SD) & $124( \pm 22)$ & $125( \pm 23)$ & n.s \\
\hline Diastolic Blood Pressure, mmHg (SD) & $80( \pm 12)$ & $80( \pm 10)$ & n.s \\
\hline Homocysteine, $\mu \mathrm{mol} / \mathrm{l}$ (range) & $10.6(5-35)$ & $11.5(6-18)$ & n.s \\
\hline CRP, mg/l (range) & $3.5(1-10)$ & $6.8(0-40)$ & n.s \\
\hline
\end{tabular}


difference in C-reactive protein in primary RP $(2 \mathrm{mg} / \mathrm{l}, \mathrm{IQR} 2$ to $3.5 \mathrm{mg} / \mathrm{l})$ and secondary RP ( $3 \mathrm{mg} / \mathrm{l}$, IQR 2 to $6 \mathrm{mg} / \mathrm{l})$.

Table 3. Nailfold Capillaroscopy in Patients with Primary and Secondary RP

\begin{tabular}{|c|c|c|}
\hline & Primary $(\mathbf{n}=\mathbf{6 0})$ & Secondary $(\mathbf{n}=\mathbf{2 1})$ \\
\hline \hline Capillary turquosity, $\mathrm{n}(\%)$ & $35(58)$ & $18(86)$ \\
\hline Irregular architecture, $\mathrm{n}(\%)$ & $1(2)$ & $12(60)$ \\
\hline Giant capillaries, $\mathrm{n}(\%)$ & $0(0)$ & $6(29)$ \\
\hline Hemorrhages, $\mathrm{n}(\%)$ & $0(0)$ & $8(38)$ \\
\hline
\end{tabular}

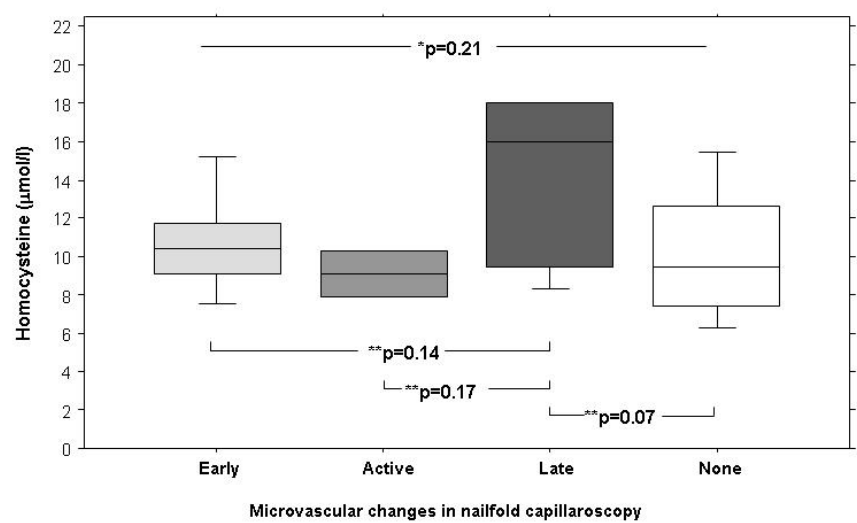

Fig. (2). Box plots of homocystein plasma serum levels in patients with Raynaud phenomenon in relation to microvascular patterns. * P-values refers to Kruskall Wallis test and ** to Bonferroni Dunn corrections (significance level $<0.0001$ ).

\section{Homocysteine Plasma Level and Morphology of Nailfold Capillaries in Secondary RP}

Median Hcy plasma level was not different in patients with $(10.4 \mu \mathrm{mol} / 1$, IQR 9 to $14.5 \mu \mathrm{mol} / \mathrm{l})$ and without microangiopathy $(9.5 \mu \mathrm{mol} / 1$, IQR 7.5 to $12.7 \mu \mathrm{mol} / 1$, $\mathrm{p}=0.09$ ). However, there were also no significant differences of median Hcy plasma levels when analyzed for the presence or absence of irregular architecture $(10.4 \mu \mathrm{mol} / \mathrm{l}$, IQR 8.6 to $15.2 \mu \mathrm{mol} / 1$ versus $10 \mu \mathrm{mol} / 1 \mathrm{IQR} 7.5$ to $12.6 \mu \mathrm{mol} / 1, \mathrm{p}=0.3$ ) or for giant capillaries $(16 \mu \mathrm{mol} / \mathrm{l}$, IQR 10.7 to $18 \mu \mathrm{mol}$ versus $9.9 \mu \mathrm{mol} / \mathrm{l}$, IQR 7.7 to $12.6 \mu \mathrm{mol}, \mathrm{p}=0.07$ ), both specific features in connective tissue diseases [20]. No difference was found when microvascular changes were summarized as proposed by Cutolo et al. Hcy was $9.5 \mu \mathrm{mol} / \mathrm{l}$ (IQR 7.5 to $12.6 \mu \mathrm{mol} / \mathrm{l})$ in patients with "none" patterns, $10.2 \mu \mathrm{mol} / 1$ (IQR 9.1 to $10.4 \mu \mathrm{mol} / \mathrm{l}$ ) in patients with "early" pattern, in patients with "active" pattern $9.1 \mu \mathrm{mol} / \mathrm{l}$ (IQR 8.5 to $9.7 \mu \mathrm{mol} / \mathrm{l}$ ), and in "late" pattern $14.1 \mu \mathrm{mol} / \mathrm{l}$ ( IQR 10 to $16.8 \mu \mathrm{mol} / 1)$.

\section{Homocysteine Plasma Levels in Patients with Primary and Secondary Raynaud Phenomenon and Cardiovas- cular Risk Factors}

There was no difference of median Hcy level between patients with and without cardiovascular risk factors $(9.8 \mu \mathrm{mol} / \mathrm{l}$, IQR 7.3 to $11.9 \mu \mathrm{mol} / 1$ versus $10 \mu \mathrm{mol} / \mathrm{l}$, IQR 8.2 to $13.2 \mu \mathrm{mol} / \mathrm{l})$. Hcy level was not different in patients with one or more cardiovascular co-morbidities (10.0 mol/l, IQR
8.0 to $12.6 \mu \mathrm{mol} / \mathrm{l})$ compared to patients without $(10.0 \mu \mathrm{mol} / \mathrm{l}$, IQR 8.2 to $12.6 \mu \mathrm{mol} / 1, \mathrm{p}=0.4$ ).

\section{DISCUSSION}

The multiple pathways in which Hcy affects vascular integrity and endothelial functional properties suggest a possible role of Hcy in RP and atherosclerosis..

Primary Raynaud phenomenon and Hcy: In the present study no significant difference of Hcy in primary $(10.6 \mu \mathrm{mol} / \mathrm{l})$ and secondary $(11.5 \mu \mathrm{mol} / \mathrm{l}) \mathrm{RP}$ had been found. Levy et al. demonstrated in 20 patients with primary and secondary RP that plasma Hcy level was higher than in healthy controls [15]. Interestingly, in his study patients with primary RP had a higher Hcy than those with secondary RP $(15.5 \mu \mathrm{mol} / 1$ and $11.6 \mu \mathrm{mol} / 1)$. However the value for secondary RP was almost the same in our study $(11.5 \mu \mathrm{mol} / 1)$

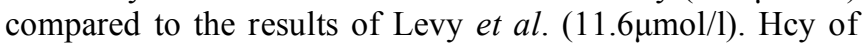
$15.5 \mu \mathrm{mol} / 1$ found in primary RP is rather high for these otherwise healthy subjects and explains the significant difference between healthy controls and patients with RP in the study of Levy. Furthermore, the presences of cardiovascular risk factors or diseases that may cause elevated Hcy were not reported in the study of Levy. In two other studies patients with primary RP had Hcy levels of 7.8 and $9.8 \mu \mathrm{mol} / 1$ respectively, whereas in one study Hcy was even higher in controls $(9.4 \mu \mathrm{mol} / \mathrm{l})$ than in primary RP $(7.8 \mu \mathrm{mol} / \mathrm{l})[16]$. These values correspond well with our result in primary RP $(10.6 \mu \mathrm{mol} / 1)[12,16]$. Therefore, from the current data including our study there is not sufficient evidence that in patients with primary RP Hcy is significantly elevated.

Secondary Raynaud phenomenon and Hcy: In our patient group microvascular changes and Hcy levels in secondary RP did not correlate as previously reported. Caramaschi et al. reported higher values of Hcy in patients with systemic sclerosis $(11.8 \mu \mathrm{mol} / \mathrm{l})$ than in healthy controls. They found that the level of Hcy correlated to the severity of microangiopathy in nailfold capillaroscopy [19]. In our study population the number of patients with systemic sclerosis was rather low, whereas Caramaschi included only patients with systemic sclerosis. Again, the value of Hcy in the study of Caramaschi was similar to the value we had in our patient group (11.8 versus $11.5 \mu \mathrm{mol} / \mathrm{l})$ with secondary RP. Other published data on Hcy in secondary RP are in the same range $(10.6$ to $13.1 \mu \mathrm{mol} / \mathrm{l})[13,15,16,22]$. In the general population up to $10 \%$ have Hcy values of more than $10 \mu \mathrm{mol} / \mathrm{l}$. The controversial results of the rather small studies comparing Hcy in patients with RP and healthy controls depend mostly on the differences in the control group which range from 5.9 to $10.7 \mu \mathrm{mol} / \mathrm{l}$. Cheng et al. compared Hcy levels in patients with SLE either with or without RP [13]. He found higher Hcy levels in SLE patients with RP $(11.7 \mu \mathrm{mol} / 1)$ but not in those without RP $(8.3 \mu \mathrm{mol} / \mathrm{l})$ compared to controls $(8.0 \mu \mathrm{mol} / \mathrm{l})$. It might be that in autoimmune diseases the elevation of Hcy represents an additional risk factor for developing vascular damage. Data proving this assumption are very scarce. We hypothesised that in patients with elevated plasma Hcy and RP inflammation or cardiovascular risk factors might promote microvascular alterations. In our study there were no differences of CRP or cholesterol levels between primary 
and secondary RP. Furthermore, pathological features of nailfold capillaries did not correlate with CRP or any cardiovascular risk factors. Despite some short comings of this study as small simple size, cross section data with only one time measurement and potential sample bias, it still adds to the scarce data available on this topic and rather supports that Hcy level are probably not of major relevance in Raynaud phenomenon.

In conclusion, we did not find any difference in Hcy levels in patients with primary or secondary RP. Neither Hcy nor cardiovascular risk factors nor comorbidities were associated with microvascular changes.

Prospective studies would be needed to further investigate whether Hcy is involved in the progression of micro- and macrovascular complications in different systemic diseases.

\section{ACKNOWLEDGEMENT}

None declared.

\section{CONFLICT OF INTEREST}

There is no conflict of interest by any of the investigators performing the study.

\section{REFERENCES}

[1] Bagi Z, Ungvari Z, Szollar L, Koller A. Flow-induced constriction in arterioles of hyperhomocysteinemic rats is due to impaired nitric oxide and enhanced thromboxane a(2) mediation. Arterioscler Thromb Vasc Biol 2001; 21: 233-7.

[2] Constans J, Conri C. Circulating markers of endothelial function in cardiovascular disease. Clin Chem Acta 2006; 368: 33-47.

[3] Woo KS, Chook P, Lolin YI, et al. Hyperhomocyst(e)inemia is a risk factor for arterial endothelial dysfunction in humans. Circulation 1997; 96: 2542-4.

[4] Tyagi SC, Smiley LM, Mujumdar VS, Clonts B, Parker JL. Reduction-oxidation (redox) and vascular tissue level of homocyst(e)ine in human coronary atherosclerotic lesions and role in extracellular matrix remodeling and vascular tone. Mol Cell Biochem 1998; 181: 107-16.

[5] Sydow K, Schwedhelm E, Arakawa N, et al. Adma and oxidative stress are responsible for endothelial dysfunction in hyperhomocyst(e)inemia: Effects of l-arginine and b vitamins. Cardiovasc Res 2003; $57: 244-52$.

[6] Stuhlinger MC, Oka RK, Graf EE, et al. Endothelial dysfunction induced by hyperhomocyst(e)inemia: Role of asymmetric dimethylarginine. Circulation 2003; 108: 933-8.
[7] Poddar R, Sivasubramanian N, DiBello PM, Robinson K, Jacobsen DW. Homocysteine induces expression and secretion of monocyte chemoattractant protein-1 and interleukin-8 in human aortic endothelial cells: Implications for vascular disease. Circulation 2001; 103: 2717-23.

[8] Lambert J, van den Berg M, Steyn M, et al. Familial hyperhomocysteinaemia and endothelium-dependent vasodilatation and arterial distensibility of large arteries. Cardiovasc Res 1999; 42: 743-51.

[9] Giugliano D, Ceriello A, Paolisso G. Oxidative stress and diabetic vascular complications. Diabetes Care 1996; 19: 257-67.

[10] Ceriello A. Oxidative stress and diabetes-associated complications. Endocr Pract 2006; 12 (Suppl 1): 60-2.

[11] Sydow K, Hornig B, Arakawa N, et al. Endothelial dysfunction in patients with peripheral arterial disease and chronic hyperhomocysteinemia: Potential role of adma. Vasc Med 2004; 9: 93-101.

[12] al-Awami M, Schillinger M, Maca T, et al. Homocysteine levels in patients with raynaud's phenomenon. Vasa 2002; 31: 87-90.

[13] Cheng TT, Chiu CK. Elevated homocysteine levels in patients with raynaud's phenomenon secondary to systemic lupus erythematosus. Clin Rheumatol 2002; 21: 251-4.

[14] Danese S, Candelli M, Gasbarrini A. Elevated homocysteine levels in patients with raynaud's syndrome. J Rheumatol. 2000; 27: 2051.

[15] Levy Y, George J, Langevitz P, et al. Elevated homocysteine levels in patients with raynaud's syndrome. J Rheumatol 1999; 26: 23835.

[16] Marasini B, Casari S, Bestetti A, et al. Homocysteine concentration in primary and systemic sclerosis associated raynaud's phenomenon. J Rheumatol 2000; 27: 2621-3.

[17] Stanger O, Herrmann W, Pietrzik K, et al. Dach-liga homocystein (german, austrian and swiss homocysteine society): Consensus paper on the rational clinical use of homocysteine, folic acid and bvitamins in cardiovascular and thrombotic diseases: Guidelines and recommendations. Clin Chem Lab Med 2003; 41: 1392-403.

[18] Gao XM, Wordsworth P, McMichael AJ, et al. Homocysteine modification of hla antigens and its immunological consequences. Eur J Immunol 1996; 26: 1443-50.

[19] Caramaschi P, Volpe A, Canestrini S, et al. Correlation between homocysteine plasma levels and nailfold videocapillaroscopic patterns in systemic sclerosis. Clin Rheumatol 2007; 26: 902-7.

[20] Meli M, Gitzelmann G, Koppensteiner R, Amann-Vesti BR Predictive value of nailfold capillaroscopy in patients with raynaud's phenomenon. Clin Rheumatol 2006; 25: 153-8.

[21] Cutolo M, Pizzorni C, Tuccio M, et al. Nailfold videocapillaroscopic patterns and serum autoantibodies in systemic sclerosis. Rheumatology (Oxford) 2004; 43: 719-26.

[22] Czupryniak A, Kaluzynska A, Nowicki M, Wiecek B, Bald E, Owczarek D. Raynaud's phenomenon and endothelial dysfunction in end-stage renal disease patients treated with hemodialysis. Kidney Blood Press Res 2005; 28: 27-31. 